\title{
Flebotomíneo em fragmentos de Mata Atlântica na Região Metropolitana do Recife, PE
}

\section{Phlebotomine sandflies in fragments of rain forest in Recife, Pernambuco State}

\author{
Dílvia Ferreira Silva $^{1}$ e Simão Dias Vasconcelos ${ }^{2}$
}

\begin{abstract}
RESUM0
Foi realizada uma investigação sobre a distribuição da fauna flebotomínica em 4 fragmentos da Mata Atlântica na Região Metropolitana do Recife. Consistiu na captura de insetos adultos com auxilio de armadilhas luminosas CDC. 1.173 espécimes distribuídos em 11 espécies de Lutzomyia: Lutzomyia evandroi, Lutzomyia choti, Lutzomyia walkeri, Lutzomyia umbratilis, Lutzomyia brasiliensis, Lutzomyia sordellii, Lutzomyia claustrei, Lutzomyia wellcomei, Lutzomyia fluviatilis, Lutzomyia furcata e Lutzomyia aragaoi.
\end{abstract}

Palavras-chaves: Lutzomyia. Flebotomíneos. Mata Atlântica. Fragmentos.

\begin{abstract}
An investigation was conducted into the distribution of sandfly fauna in 4 fragments of Atlantic forest in the Metropolitan Area of Recife. It consisted of the capture adult insects using CDC light traps. A total of 1,173 specimens were distributed in 11 species of Lutzomyia: Lutzomyia evandroi, Lutzomyia choti, Lutzomyia walkeri, Lutzomyia umbratilis, Lutzomyia brasiliensis, Lutzomyia sordellii, Lutzomyia claustrei, Lutzomyia wellcomei, Lutzomyia fluviatilis, Lutzomyia furcata e Lutzomyia aragaoi.
\end{abstract}

Key-words: Lutzomyia. Sandflies. Rain Forest. Fragment.

No Nordeste do Brasil existe uma grande riqueza de espécies de flebotomíneos. Estados como Maranhão, Ceará e Bahia apresentam uma fauna bastante diversificada, constituída por um grande número de espécies ${ }^{1315}$.

Em Pernambuco, a fauna flebotomínica de Lutzomyia ainda é pouco estudada quanto à diversidade e distribuição das espécies. Os dados disponíveis relatam 0 encontro de algumas espécies no município de Igarassu e na Região Metropolitana do Recife $^{1012}$. Na Zona da Mata há apenas ocorrência de algumas espécies em área endêmica da leishmaniose tegumentar ${ }^{34}$, não sendo possível determinar com mais clareza a composição da fauna flebotomínica existente naquela área. Entre os anos de 1990 -1999 foram registrados para o Estado de Pernambuco 7.616 casos, dos quais $6.308(82,8 \%)$ eram de leishmaniose tegumentar americana e 1.308 (17,2\%) leishmaniose visceral. 0 número de homens afetados pelas leishmanioses foi superior ao encontrado em mulheres ${ }^{14}$.

Este trabalho tem como objetivo investigar a distribuição dos flebotomíneos em fragmentos da Mata Attântica localizadas na Região Metropolitana do Recife.
Área de estudo. Para este estudo, foram escolhidas 4 áreas formada por fragmentos de Mata Attântica localizadas em diferentes pontos da Região Metropolitana do Recife. Pernambuco compreende uma área de $98.937 \mathrm{~km}^{2}$ e uma população de 7,5 milhões de habitantes. 0 clima é tropical, com índice pluviométrico de $2000 \mathrm{~mm}$ no litoral e $600 \mathrm{~mm}$ no interior. AEstação Ecológica do Tapacurá ( 34으' W; 08007' S) , município de São Lourenço da Mata, ocupa uma área de 776ha, sendo 348 ha referente ao lago formado pelo represamento do rio Tapacurá. A área é ocupada por floresta ombrófila aberta. A Mata do Curado ( 34057' W ; 8004' S) , município de Recife, com cerca de 43,40 ha. situada na 7a Região Militar do Comando Militar do Nordeste. Área de formação pioneira, apresentando estratos arbóreos, arbustivos e herbáceos. Mata de Dois Irmãos ( 34056'00'W; 800'00'S) , próxima a Universidade Federal Rural de Pernambuco, possui área de 387,42ha com estrato arbóreo denso formando um dossel que atinge $20 \mathrm{~m}$ de altura, estrato arbustivo escasso e herbáceo presente em áreas semi-abertas com maior penetração da luz. 0 Refúgio Ecológico Charles Darwin (34ํㄱ'25”W; 7²4'37”S) no município de Igarassu,

1. Laboratório de Leishmaniose e Doença de Chagas do Centro de Pesquisa em Ciência da Saúde do Instituto Nacional de Pesquisas da Amazônia, Manaus, AM. 2. Departamento de Zoologia do Centro de Ciências Biológicas da Universidade Federal de Pernambuco, Recife, PE.

Endereço para correspondência: Drª Dílvia Ferreira da Silva. Laboratório de Leishmaniose e Doença de Chagas /INPA. Av. André Araújo 2936, Petrópolis, Caixa Postal 478, 69011-970, Manaus, AM.

Tel:55 92 643-3065

e-mail: dilvia@inpa.gov.br

Recebido para publicação e 10/3/2004

Aceito em 3/3/2005 
litoral norte de Pernambuco, possui área de 60ha com mata úmida, remanescente da primitiva floresta atlântica costeira.

Captura dos flebotomíneos. As coletas foram realizadas no período de um ano compreendido entre outubro/1999 e setembro/2000. Para captura, foram utilizadas armadilhas CDCa uma altura de 1 metro com períodos de 12 horas, dois dias em cada área de mata, perfazendo um total de 1.152 horas. Os insetos coletados foram colocados em álcool 70\% e levados para identificação no Laboratório de Invertebrados Terrestres do Departamento de Zoologia, Universidade Federal de Pernambuco. Para identificação, foi utilizada a chave de Young \& Duncan ${ }^{16}$.

No período de 12 meses (outubro/1999 a setembro/2000) foram coletados um total de 1.173 flebotomíneos nas quatro áreas de mata da Região Metropolitana do Recife. Estes flebotomíneos estão distribuídos em 11 espécies pertencentes ao gênero Lutzomyia França. As espécies deste gênero são L evandroi (Costa Lima \& Antunes 1936), L choti (Floch \& Abonnenc 1944), L walkeri (Newstead 1914), L umbratilis (Ward \& Fraiha 1977), L brasiliensis (Costa Lima 1932), L sordellii ( Shannon \& Del Ponte 1927) , L claustrei (Abonnenc, Léger \& Fauran 1979), L wellcomei (Fraiha, Shaw \& Lainson 1971), L fluviatilis (Floch \& Abonnenc 1944), L furcata (Mangabeira 1941) e L aragaoi (Costa Lima 1932) (Tabela 1).

Dentre os espécimes coletados 678 (58\%) fêmeas e 495 (42\%) machos, ocorrendo uma diferença significante na abundância entre ambos os sexos $(p<0,05)$ (Figura 1$)$.

Os resultados deste trabalho apresentaram a fauna flebotomínica encontrada em 4 áreas compostas por fragmentos da Mata Atlântica localizadas na Região Metropolitana do Recife. Das 11 espécies capturadas, apenas Lutzomyia furcata e

Tabela 1- Número total de espécies de flebotomíneos coletados em área de mata da Estação Ecológica do Tapacurá, Curado, Dois Irmãos e Refúgio Ecológico Charles Darwin, na Região Metropolitana do Recife-PE.

\begin{tabular}{|c|c|c|c|c|c|}
\hline \multirow[b]{2}{*}{ Espécies } & \multicolumn{5}{|c|}{ Áreas de Mata } \\
\hline & Tapacura & Curado & Dois Irmãos & Charles Darwin & total \\
\hline Lutzomyia aragaoi & 173 & 2 & - & - & 175 \\
\hline Lutzomyia braziliensis & 66 & - & - & - & 66 \\
\hline Lutzomyia choti & - & - & - & 307 & 307 \\
\hline Lutzomyia claustrei & - & - & - & 34 & 34 \\
\hline Lutzomyia evandroi & - & 96 & 133 & 83 & 312 \\
\hline Lutzomyia fluviatilis & - & - & 10 & - & 10 \\
\hline Lutzomyia furcata & - & 19 & 4 & - & 23 \\
\hline Lutzomyia sordellii & - & 32 & - & 16 & 48 \\
\hline Lutzomyia umbratilis & - & - & 89 & - & 89 \\
\hline Lutzomyia walkeri & - & 92 & - & - & 92 \\
\hline Lutzomyia wellcomei & - & - & - & 17 & 17 \\
\hline Total & 239 & 241 & 236 & 457 & 1.173 \\
\hline
\end{tabular}

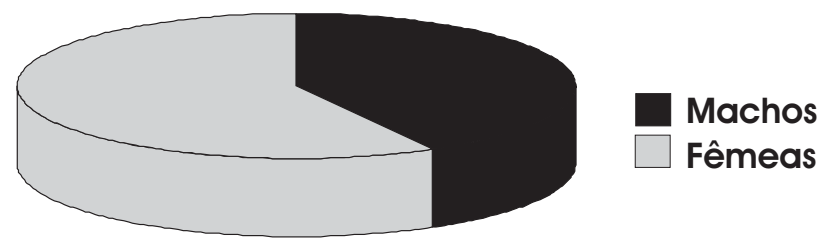

Figura 1- Percentual de flebotomíneos machos e fêmeas coletados nas quatro áreas de mata da Região Metropolitana do Recife, PE.
L evandroi já haviam sido citadas em levantamentos anteriores conduzidos nesta região para as áreas de Dois Irmãos e Igarassu ${ }^{678}$. Por outro lado, as espécies L.schereiberi, L. oswaldoi, L intermedia, L shannoni e L squamiventris registradas nos citados estudos, conduzidos há cerca de 20 anos, não foram capturadas na presente pesquisa. Podemos inferir que o horário de coleta e as armadilhas utilizadas podem ter influenciado na captura de diferentes espécies.

Arazão total fêmeas/machos das espécies coletadas encontrase próxima de 2:1. Aguiar et $\mathrm{al}^{12}$, acreditam que coletas com armadilha luminosa possa atrair um maior número de machos, visto que estes formam um agregado com o propósito de acasalamento. Em nossas coletas foi observado que 0 número de machos foi inferior ao número de fêmeas, não corroborando com o pressuposto observado pelos autores acima citado.

Verificou-se que a Estação Ecológica do Tapacurá apresentou o menor índice de diversidade, o que pode estar relacionado ao nível de devastação local com modificação da sua flora original. Na mata do Curado foi observado o maior índice de diversidade, acredita-se que a conservação da mata primitiva neste local resulte na permanencia dos mamíferos silvestres nos quais os flebotomíneos realizam o repasto sanguineo.

Das espécies encontradas, duas são importantes para a saúde pública, L umbratilis e L wellcomei. A primeira, considerada vetor silvestre da Leishmania (Viannia) guyanensis no norte do país, aparentemente prefere praticar hematofagia na copa das árvores onde se encontram seus hospedeiros naturais ${ }^{515}$, Ready et al ${ }^{9}$ afirmaram que pessoas que realizaram desmatamentos em seus lotes para estabelecer suas moradias em áreas próximas a Manaus contraíram leishmaniose quando picadas pela L umbratilis.

Ready et $\mathrm{al}^{10}$ registraram a ocorrência de L wellcomei fora da Bacia Amazônica, em ambiente florestal no Estado do Ceará. É provável que a larga distribuição de Leishmania (Viannia) braziliensis nos Estados da Paraíba, Bahia e Ceará resultem da transmissão por L wellcomei ${ }^{41015}$, Rebêlo et al $^{1213}$ ao relatarem a presença destas espécies no Maranhão, sugerem que a retirada da cobertura vegetal da Ilha de São Luís pode ter favorecido 0 deslocamento de animais silvestres no qual os flebotomíneos fazem 0 repasto, conduzindo estes insetos a procurarem fontes alternativas de alimento, ocorrendo com isso surtos epidêmicos da leishmaniose visceral e tegumentar americana.

Embora L umbratilis e L wellcomei tenham sido capturadas no Refúgio Ecológico Charles Darwin e na Mata de Dois Irmãos, não se pode responsabilizá-las como vetores da Leishmania (Viannia) braziliensis e Leishmania (Viannia) guyanensis em Pernambuco devido à ausência de análise destes insetos encontrados com parasitos, relatando a ocorrência e possíveis implicações na transmissão da leishmaniose tegumentar. Esta carência também se deve à falta de estudos específicos demonstrando o envolvimento de diferentes espécies de Leishmania na infecção da leishmaniose tegumentar americana. Até 0 momento, apenas L whitmani tem sido incriminada como responsável pela transmissão da leishmaniose cutânea e L longipalpis pela leishmaniose visceral para este estado. 
Os resultados deste trabalho ampliam 0 conhecimento acerca da distribuição dos flebotomíneos em áreas de mata localizadas na Região Metropolitana do Recife. 0 registro nessas matas de duas espécies incriminadas na transmissão da leishmaniose em outra região, é motivo de preocupação, justificando maiores investigações sobre este fenômeno.

\section{REFERÊNCIAS BIBLIOGRÁFICAS}

1. Aguiar GM, Medeiros WM, De Marco TS, Santos SC, Gambardella S. Ecologia dos flebotomíneos da Serra do Mar, Itaguaí, Estado do Rio de Janeiro, Brasil. Caderno de Saúde Pública 12:195-206, 1996.

2. Aguiar GM, Vilela ML, Schuback PD, Soucasaux T, Azevedo ACR. Aspectos da ecologia dos flebótomos do Parque Nacional da Serra dos Órgãos, Rio de Janeiro. IV. Frequência mensal em armadilhas luminosas (Diptera, Psychodidae, Phlebotominae). Memórias do Instituto Oswaldo Cruz 80:465-482, 1985

3. Brandão-Filho SP, Brito MEF, Martins CAP, Sommer IB, Valença HF, Almeida FA, Gomes J. Leishmaniose tegumentar americana em centro de treinamento militar localizado na zona da Mata de Pernambuco, Brasil. Revista da Sociedade Brasileira de Medicina Tropical 31:575-578, 1998.

4. Brandão-Filho SP, Carvalho FG, Brito MEF, Almeida FA, Nascimento LA. American cutaneous leishmaniasis in Pernambuco, Brazil: Ecoepidemiological aspects in "Zona da Mata" region. Memorias do Instituto Oswaldo Cruz 89:445-449, 1994.

5. Grimaldi G, Tesh RB, Mcmahon-Pratt D. A review of the geographic distribution and epidemiology of leishmaniasis in the New World. The American Journal of Tropical Medicine and Hygiene 41:687-725, 1989.

6. Lucena DT, Oliveira MHCC, Leal MCA. Variação mensal dos flebotomíneos (Diptera:Psychodidae) de Igarassu, PE. Caderno Ômega da Universidade Federal Rural de Pernambuco - Série Biologia 1:19-27, 1984.

7. Oliveira MHC, Leal MC, Nascimento AMLC. Variação mensal dos flebotomíneos (Diptera:Psychodidae) de Dois Irmãos- açude da Prata. Caderno Ômega Universidade Federal Rural de Pernambuco 1:59-65, 1984.
8. Oliveira MHC, Lucena DT, Leal MC, Nascimento AMLC. Flebotomíneos gênero Lutzomyia França, 1924, da Região Metropolitana no Recife (Diptera-Psychodidae). Anais da Universidade Federal Rural de Pernambuco 2:45-82, 1978.

9. Ready PD, Arias JR, Freitas RA. A pilot to control Lutzomyia umbratilis (Diptera:Psychodidae), the major vector of Leishmania brasiliensis guyanensis, in a peri-urban rainforest of Manaus, Amazonas state, Brazil. Memórias do Instituto Oswaldo Cruz 80:27-36, 1985.

10. Ready PD, Ribeiro AL, Lainson R, Alencar JE, Shaw JJ. Presence of Psychodopygus wellcomei (Diptera: Psychodidae), a proven vector of Leishmania braziliensis braziliensis, in Ceará State. Memórias do Instituto Oswaldo Cruz 78: 235-236, 1983.

11. Rebêlo JMM, Mendes WA, Costa JML, Cavaleiro N. Lista preliminar das espécies do gênero Lutzomyia França, 1924 (Psychodidae, Phlebotominae) do Estado do Maranhão, Brasil. Caderno de Saúde Pública 12:545-549, 1996

12. Rebêlo JMM, Araújo JAC, Carvalho ML, Barros VLL, Silva FS, Oliveira ST. Flebótomos (Diptera:Phlebotominae) da Ilha de São Luis, Zona do Golfão Maranhense, Brasil. Revista da Sociedade Brasileira de Medicina Tropical 32: 247-253, 1999.

13. Rebêlo JMM, Oliveira ST, Barros VL, Silva FS, Costa JML, Ferreira LA, Silva AR. Phlebotominae (Díptera:Psychodidae) de Lagoas, município de Buriticupu, Amazônia Maranhense. I - Riqueza e abundancia relativa das espécies em área de colonização recente. Revista da Sociedade Brasileira de Medicina Tropical 33: 11-19, 2000.

14. Silva DF, Vasconcelos SD. A ten year (1990-1999) survey on leishmaniasis incidence in Pernambuco state, northeastern Brazil. Revista de Patologia Tropical 32:51-61, 2002.

15. Sherlock IA, Maia H, Dias-Lima AG. Resultados preliminares de um projeto sobre a ecologia dos flebotomíneos vetores da leishmaniose tegumentar no Estado da Bahia. Revista da Sociedade Brasileira de Medicina Tropical 29: 207-214, 1996.

16. Young DG, Duncan MA. Guide to the identification and geographic distribution of Lutzomyia sand flies in Mexico, the west Indies, Central and South American (Diptera:Psychodidae). Memories American Entomology Institute 54: 1-881, 1994 